\title{
POLITRAUMATIZADOS COM TRAUMA CRANIENCEFÁLICO GRAVE: IMPORTÂNCIA DAS LESÕES ABDOMINAIS ASSOCIADAS
}

\author{
SEVERE BLUNT HEAD TRAUMA: THE RELEVANCE OF ASSOCIATED \\ ABDOMINAL LESIONS
}

\author{
José Gustavo Parreira, TCBC-SP ${ }^{1}$ \\ Raul Coimbra, TCBC-SP ${ }^{2}$ \\ Samir Rasslan, TCBC-SP ${ }^{3}$ \\ Dan Enger Ruiz ${ }^{4}$
}

\begin{abstract}
RESUMO: Objetivo: Analisar as lesões abdominais dos politraumatizados com trauma craniencefálico (TCE) grave. Método: Realizou-se avaliação retrospectiva dos prontuários das vítimas de TCE no período de 1996 a 1997. Foram selecionados para o estudo os com Escala de Coma de Glasgow (ECG) $\leq 8$ à admissão. Cinquenta e dois politraumatizados foram incluídos, com média etária de 37 anos, sendo 44 do sexo masculino. Avaliamos os dados demográficos, índices de trauma (RTS, ISS), exames complementares, tratamento e evolução. A análise estatística foi realizada com os testes qui quadrado, t de Student, exato de Fisher e cálculo do odds ratio, considerando-se $\mathrm{p} \leq 0,05$ como estatisticamente significante. Resultados: A média do RTS foi 5,18 e do ISS foi 27. O mecanismo de trauma mais freqüente foi o atropelamento, em 25 casos. Nenhum destes doentes teve diagnóstico de lesão abdominal apenas pelo exame físico. Quarenta e quatro doentes foram avaliados por lavagem peritoneal diagnóstica e 8 por ultra-sonografia ou tomografia computadorizada abdominal. Oito doentes (15\%) apresentaram lesões abdominais, havendo 5 lesões hepáticas, 4 esplênicas e uma em cólon. Realizou-se 17 craniotomias e 8 laparotomias (4 não terapêuticas). As lesões abdominais estiveram relacionadas à pressão arterial sistólica menor que $90 \mathrm{mmHg}(\mathrm{p}=0,05)$ à admissão e à presença de lesões ortopédicas de tratamento operatório $(\mathrm{p}<0,001)$. A letalidade foi $71 \%$. Conclusão: Uma parcela considerável dos traumatizados com TCE grave apresenta lesões abdominais e o exame físico contribui pouco para o seu diagnóstico. Justifica-se a investigação objetiva do abdome com métodos complementares em todos estes casos.
\end{abstract}

Descritores: Trauma craniencefálico; Trauma abdominal; Laparotomia; Diagnóstico.

\section{INTRODUÇÃO}

A melhora no atendimento pré-hospitalar, o aumento da violência urbana e dos acidentes automobilísticos são responsáveis pela complexidade crescente dos traumatizados admitidos em hospitais ${ }^{1}$. Freqüentemente os doentes apresentam associação de lesões graves em segmentos corpóreos distintos.

No politraumatizado com trauma cranioencefálico (TCE) grave pode ser difícil o diagnóstico de lesões abdo- minais. Sabe-se que $40 \%$ dos hemoperitônios são assintomáticos, a acurácia do exame físico abdominal não ultrapassa $65 \%$ e a diminuição do nível de consciência dificulta ainda mais o exame clínico ${ }^{2-6}$. Essas limitações resultam em mortes evitáveis após traumatismos por lesões abdominais não diagnosticadas, mesmo considerando-se que a necessidade real de laparotomias por trauma abdominal fechado não ultrapasse $5 \%{ }^{6-8}$. Por outro lado, não deve ocorrer retardo no tratamento de lesões neurocirúrgicas, pois isto interfere definitivamente no prognóstico ${ }^{5,9}$.

1. Médico Assistente do Serviço de Emergência.

2. Professor Associado - Universidade da Califórnia, San Diego, EUA.

3. Professor Titular. Diretor do Serviço de Emergência.

4. Residente de Cirurgia.

Recebido em 18/08/2000

Aceito para publicação em 26/06/2001

Trabalho realizado no Serviço de Emergência do Departamento de Cirurgia da Faculdade de Ciências Médicas da Santa Casa de São Paulo. 
A tomografia axial computadorizada (TAC) de crânio é o melhor exame diagnóstico no TCE. Para a avaliação abdominal são utilizados vários métodos complementares como a ultra-sonografia (US), a tomografia axial computadorizada e a lavagem peritoneal diagnóstica (LPD), cada qual com suas vantagens e desvantagens ${ }^{8,10,11}$.

Mesmo com os recursos atualmente disponíveis, em algumas situações podem haver dúvidas quanto ao diagnóstico de uma lesão abdominal e a necessidade de tratamento operatório.

O objetivo deste estudo é avaliar as lesões abdominais dos politraumatizados admitidos com TCE grave.

\section{MÉTODO}

Realizamos análise retrospectiva através de coleta de dados dos protocolos de trauma e dos prontuários dos traumatizados admitidos no período de janeiro de 1996 a julho de 1997. Incluímos no grupo de estudo doentes com TCE fechado e Escala de Coma de Glasgow (ECG) menor ou igual a oito à admissão (definido como grave).

Foram avaliados os dados de 52 traumatizados, 44 deles do sexo masculino, com média etária de 37 anos. O mecanismo de trauma mais frequiente foi o atropelamento, em 25 casos (48\%) (Tabela 1).

Foram analisados mecanismo de trauma, dados vitais e índices de trauma à admissão, métodos de avaliação abdominal, lesões encontradas, laparotomias, craniotomias, complicações e óbitos. As lesões associadas foram estratificadas pelo Abbreviated Injury Scale (AIS) e Organ Injury Scale (OIS) ${ }^{12,13}$. O grau de comprometimento neurológico funcional foi quantificado pela $\mathrm{ECG}^{14}$. Os índices de trauma utilizados foram o RTS e o ISS ${ }^{15-17}$. Denominamos indicadores de lesões abdominais os fatores que estatisticamente se associaram a presença de trauma em
Tabela 1

Mecanismo de trauma

\begin{tabular}{l|cc}
\hline Mecanismo Trauma & \multicolumn{2}{c}{ Número (\%) } \\
\hline Atropelamento & 25 & $(48 \%)$ \\
Acidente automobilístico & 12 & $(23 \%)$ \\
Queda & 10 & $(20 \%)$ \\
Agressão & 5 & $(9 \%)$ \\
\hline Total & 52 & $(100 \%)$ \\
\hline
\end{tabular}

vísceras abdominais. Comparamos variáveis entre os doentes com e sem estas lesões para determiná-las. Os indicadores pesquisados foram a idade, mecanismo de trauma, ECG e choque à admissão (definido como pressão arterial sistólica $<90 \mathrm{mmHg}$ ), lesões graves (AIS $\geq 3$ ) em crânio, tórax, extremidades, realização de craniotomia e fraturas de bacia. A opção pelo tratamento de lesões em vísceras parenquimatosas baseou-se na necessidade de controle do sangramento. A análise estatística foi realizada através dos testes qui quadrado, t de Student e exato de Fisher, adotando-se como estatisticamente significativos valores de $\mathrm{p}<0,05$. Calculou-se o odds ratio para cada variável.

\section{RESULTADOS}

A média do RTS foi $5,18 \pm 1,4$, a do ISS foi $27 \pm 9$ e da ECG foi 5,36 $\pm 1,6$. Oito doentes foram admitidos em choque. A avaliação abdominal foi realizada por lavagem

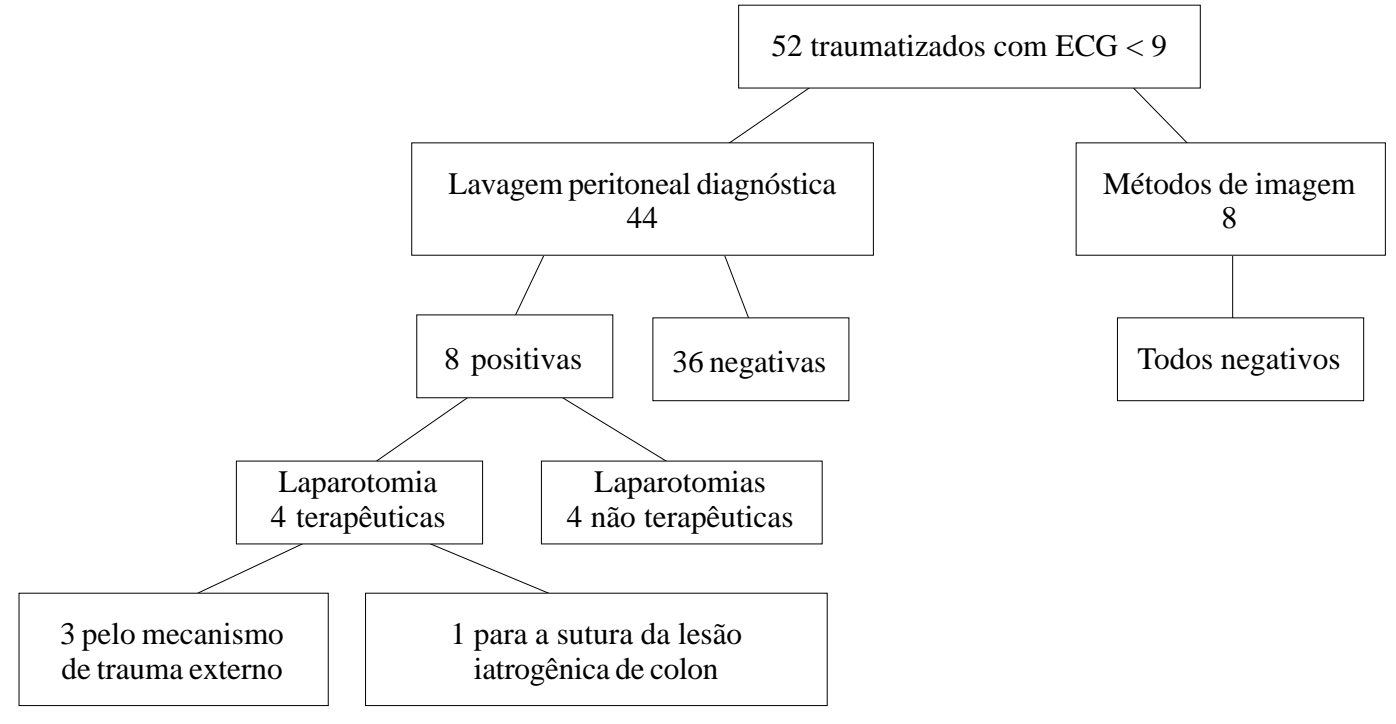

Quadro 1 - Avaliação abdominal e condutas terapêuticas. 
peritoneal diagnóstica (LPD) em 44 doentes. Destas, 8 foram positivas e 36 negativas. Não houve falso-positivos ou negativos. Ocorreu uma complicação, a perfuração do cólon pela passagem do cateter em um caso de LPD positiva. Em oito doentes foram realizados métodos de imagem (ultra-som e/ou tomografia) para a avaliação abdominal, sendo todos os exames negativos (Quadro 1).

As lesões neurológicas mais encontradas foram o brain swelling, em 19 doentes (36\%) e a lesão axonal difusa, em 17 (32\%) (Tabela 2). Foram realizadas 17 craniotomias $(32 \%)$. As indicações mais frequientes foram os hematomas extradurais (41\%), os hematomas subdurais (41\%) e as contusões (29\%). Apenas 3 traumatizados foram submetidos a craniotomia e laparotomia (Tabela 3 ).
Tabela 2

Lesões Neurológicas

\begin{tabular}{l|c|c}
\hline Lesão & $\begin{array}{c}\text { Número de } \\
\text { Doentes }\end{array}$ & $\%$ \\
\hline "Brain swelling" & 19 & 36 \\
Lesão axonal difusa & 17 & 32 \\
Hemorragia subaracnóidea & 17 & 32 \\
Hematoma subdural & 14 & 26 \\
Contusão cerebral & 13 & 25 \\
Hematoma extradural & 9 & 17
\end{tabular}

Tabela 3

Lesões encontradas, estado hemodinâmico à admissão, índices de trauma e tratamento realizado nos doentes submetidos a laparotomia exploradora.

\begin{tabular}{|c|c|c|c|c|c|c|c|c|c|c|c|c|}
\hline & Glasgow & Choque & $R T S$ & $I S S$ & $\begin{array}{l}A I S \\
C a b\end{array}$ & $\begin{array}{c}\text { AIS } \\
\text { Tórax }\end{array}$ & $\begin{array}{l}A I S \\
A b d\end{array}$ & $\begin{array}{c}A I S \\
\text { Ext }\end{array}$ & $\begin{array}{c}\text { Frat } \\
\text { Bacia }\end{array}$ & Les Abd. & Tratamento & Óbito \\
\hline 1 & 3 & S & 2,1978 & 29 & 5 & - & 2 & - & $\mathrm{N}$ & Fígado & LNT & $\mathrm{N}$ \\
\hline 2 & 8 & $\mathrm{~N}$ & 4,804 & 22 & 3 & - & 2 & 3 & $S$ & Fígado & Sutura hepática & $\mathrm{N}$ \\
\hline 3 & 6 & $\mathrm{~N}$ & 5,9672 & 13 & 3 & - & 2 & - & $\mathrm{N}$ & Baço & LNT & S \\
\hline 4 & 3 & S & 2,19 & 50 & 5 & - & 4 & 3 & $\mathrm{~N}$ & Fígado & Sutura hepática & S \\
\hline 5 & 4 & $\mathrm{~N}$ & 3,8672 & 33 & 5 & - & 2 & 2 & $\mathrm{~N}$ & Baço e fígado & LNT & $\mathrm{S}$ \\
\hline $6 *$ & 3 & S & 2,6284 & 43 & 5 & - & 3 & 3 & S & Baço & Esplenectomia & S \\
\hline $7 *$ & 6 & $\mathrm{~N}$ & 5,6764 & 45 & 5 & 4 & 2 & - & $\mathrm{N}$ & Baço e fígado & LNT & S \\
\hline $8^{*}$ & 6 & $\mathrm{~N}$ & 5,6764 & 29 & 4 & 3 & 2 & - & $\mathrm{N}$ & Colon & Sutura colon & $\mathrm{S}$ \\
\hline
\end{tabular}

* Submetidos também a craniotomia.

FratBacia: Fraturas de Bacia.

RTS: "Revised Trauma Score".

Les Abd.: Lesões abdominais.

ISS: "Injury Severity Score".

LNT: Laparotomias não terapêuticas.

AIS: "Abbreviated Injury Scale".

$S: \operatorname{sim}$

$N:$ ñ̃o

As lesões associadas com AIS $\geq 3$ ocorreram em 18 casos $(34 \%)$. Onze doentes $(21 \%)$ apresentaram lesões em extremidades, 8 (15\%) lesões em tórax e 8 (15\%) em abdome. Realizou-se 8 laparotomias, sendo 4 terapêuticas. $\mathrm{O}$ fígado esteve lesado em 5 doentes, o baço em 4 e o cólon em 1 (lesão iatrogênica pela lavagem peritoneal). O seu tratamento e os índices de trauma estão descritos na Tabela 3.

As variáveis que indicaram traumatismo abdominal foram o choque à admissão $(p=0,05)$ e as lesões ortopédicas de tratamento operatório $(\mathrm{p}<0,001)$. As lesões abdominais estiveram presentes em $42 \%$ dos doentes admitidos em choque. A instabilidade hemodinâmica à admissão acarretou um risco para a presença de lesões intracavitárias abdominais seis vezes superior ao encontrado nos estáveis hemodinamicamente. $\mathrm{O}$ risco de traumatismo abdominal aumentou 58 vezes quando havia lesões ortopédicas de tratamento operatório. As lesões abdominais estiveram presentes em $57 \%$ destes doentes.

Idade, mecanismo de trauma, ECG à admissão, presença de lesões cranianas, torácicas e extremidades com AIS $\geq 3$, fraturas de bacia ou realização de craniotomia não se relacionaram com traumatismo abdominal $(p>0,05)$ (Tabela 4).

Quarenta e seis doentes desenvolveram complicações $(88 \%)$, sendo as pulmonares mais frequentes (Tabela 5). Trinta e sete doentes morreram $(71 \%)$, sendo o TCE a principal causa (Tabela 6). A realização de laparotomia, bem como de craniotomia não se relacionou estatisticamente com maior morbidade ou letalidade (Tabela 7). Os poli- 
traumatizados submetidos a laparotomia não terapêutica não apresentaram evolução diferente quando comparados aos que não foram operados.

Tabela 4

Indicadores de lesões abdominais.

\begin{tabular}{lc}
\hline Variáveis & Valor \\
\hline Idade & $\mathrm{NS}$ \\
Mecanismo de trauma & $\mathrm{NS}$ \\
Choque à admissão & $\mathrm{p}=0,05$ \\
& (Odds ratio $=6)$ \\
Glasgow à admissão & $\mathrm{NS}$ \\
AIS crânio $\geq 3$ & $\mathrm{NS}$ \\
AIS tórax $\geq 3$ & $\mathrm{NS}$ \\
AIS extremidades $\geq 3$ & $\mathrm{NS}$ \\
Fratura de bacia & $\mathrm{NS}$ \\
Craniotomia & $\mathrm{NS}$ \\
Lesões ortopédicas de & $\mathrm{p}<0,001$ \\
trat. operatório & (Odds ratio $=58)$ \\
\hline
\end{tabular}

$N S=$ Não significante/AIS $=$ Abbreviated Injury Scale.

Tabela 5

Complicações

\begin{tabular}{l|rc}
\hline Complicação & Número (\%) \\
\hline Pneumonia & 14 & $(26)$ \\
Pneumonia aspirativa & 7 & $(13)$ \\
Diabetes insípidus & 5 & $(10)$ \\
Insuficiência renal aguda & 4 & $(8)$ \\
Perfuração do cólon (LPD) & 1 & $(2)$ \\
Tromboembolia de pulmão & 1 & $(2)$ \\
Derrame pleural & 1 & $(2)$ \\
Encefalopatia anóxica & 1 & $(2)$ \\
\hline
\end{tabular}

\section{DISCUSSÃO}

A lesão do sistema nervoso central é uma importante causa de morte nos traumatizados ${ }^{1}$. Aproximadamente $18 \%$ das vítimas de TCE morrem e este índice é pouco influenciado por lesões extracranianas, a não ser quando as mesmas são graves e associadas a traumas cranianos leves ${ }^{1}$. Nossa amostra, envolvendo apenas vítimas de TCE grave, teve uma letalidade de $71 \%$. O prognóstico esteve associado principalmente a lesão neurológica, que foi a causa de $81 \%$ das mortes.

É fundamental o diagnóstico de lesões abdominais pois a hemorragia decorrente pode agravar o dano neuro-
Tabela 6

Causas relacionadas aos 37 óbitos*

\begin{tabular}{l|c}
\hline Fatores envolvidos & $\begin{array}{c}\text { Número de } \\
\text { doentes }\end{array}$ \\
\hline Trauma craniencefálico & 30 \\
Pneumonia & 4 \\
Choque & 3 \\
Sepse & 3 \\
Contusão miocárdica & 1 \\
Tromboembolismo pulmonar & 1 \\
\hline
\end{tabular}

*Cinco doentes apresentaram mais de um fator envolvido.

Tabela 7

Influência da laparotomia ou craniotomia na letalidade

\begin{tabular}{l|c|c|c|c}
\hline Variável & Número & Vivos & Óbitos & Valor \\
\hline Laparotomia & 8 & 2 & 6 & NS \\
Craniotomia & 17 & 2 & 15 & NS \\
\hline
\end{tabular}

$\mathrm{NS}=$ não significante

lógico. Entretanto, o retardo no tratamento de lesões neurocirúrgicas em função da realização de laparotomia não terapêutica tem consequências sérias, não só pelo pior prognóstico da lesão neurológica ${ }^{18,19}$, mas também pela morbidade da laparotomia, que pode alcançar $41 \%$ (20). A necessidade de laparotomia e craniotomia simultâneas ocorreu em $6 \%$ de nossa amostra, confirmando que esta não é uma situação freqüiente ${ }^{4,5}$.

Vários estudos tentaram identificar os traumatizados com maior propensão a lesões cranianas ou abdominais, tentando direcionar a avaliação diagnóstica ${ }^{4-6,21}$. Esta análise permite separar os politraumatizados admitidos com TCE em dois grupos. O primeiro composto por jovens com taquicardia e hipotensão, que podem ter lesões torácicas, fraturas de bacia e consumo de base, cuja prioridade é a avaliação abdominal. O segundo, composto por idosos, vítimas de quedas ou trauma craniano direto, com diminuição do nível de consciência e sinais de lateralização, nos quais a prioridade é a avaliação do sistema nervoso central por tomografia de crânio. Raramente há associação de lesões cirúrgicas intracranianas e abdominais e, neste caso, a prioridade deverá ser o tratamento daquela que comprometa a vida do doente, seja o controle da lesão abdominal com sangramento ativo, seja o alívio da hipertensão intracraniana.

Mackersie et al., em 1989, relataram que as lesões abdominais são mais freqüentes nos politraumatizados admitidos em choque, com consumo de base à gasometria arterial, com lesões torácicas ou fraturas de bacia ${ }^{6}$. Em nossa análise, englobando vítimas de trauma de crânio, confirmamos apenas a relação do trauma abdominal e choque. 
Sabe-se que as lesões abdominais são frequentes nas vítimas de fraturas de bacia $^{2,8}$, mas provavelmente devido ao reduzido número destes doentes em nossa amostra, não constatamos tal relação.

No traumatizado em coma a exclusão de uma lesão abdominal deve ser preocupação constante. $\mathrm{Na}$ presença de instabilidade hemodinâmica a LPD se impõe, estando a indicação operatória na dependência do seu resultado. Entretanto, no traumatizado estável hemodinamicamente e com ECG $\leq 8$ é discutido se a LPD é o método de escolha para a avaliação abdominal ou se um exame de imagem (US ou TAC) deve ser utilizado. A LPD apresenta falsonegativos principalmente em lesões retroperitoneais como as de cólon e duodeno, e a sua sensibilidade elevada para hemoperitônio resulta em um grande número de laparotomias não terapêuticas ${ }^{8}$. Em nossa amostra, as laparotomias não terapêuticas foram freqüentes, provavelmente devido ao emprego liberal da LPD. Trata-se de um método invasivo e que pode trazer complicações, como pode ser demonstrado neste estudo, no caso da lesão iatrogênica de cólon durante o procedimento.

A ultra-sonografia tem como principal vantagem a alta sensibilidade para a detecção de líquido livre intraperitoneal. Entretanto, isto pode estar ausente em até $27 \%$ das lesões abdominais por trauma fechado ${ }^{11}$. A tomografia computadorizada tem alta sensibilidade para diagnóstico de lesões em fígado, baço e rins, mas ainda há dificuldades na detecção das lesões de intestino delgado ${ }^{8}$. Nenhum estes métodos exclui totalmente a presença de lesões de vísceras ocas $^{8,11}$. A diminuição do nível de consciência dificulta a avaliação abdominal e, portanto, o diagnóstico destas lesões, são justamente as de maior propensão a passar despercebidas na tomografia computadorizada. Esta é a principal crítica a utilização dos métodos de imagem para a avaliação abdominal dos traumatizados com TCE grave.

Há especial interesse nesta discussão pois em alguns centros o tratamento não operatório das lesões de vísceras parenquimatosas (fígado, baço e rim) pode ser uma opção para estes doentes, mesmo com diminuição do nível de consciência ${ }^{22}$. Somente a avaliação abdominal com métodos de imagem permite este tratamento. Desta forma, a indicação de tratamento não operatório em politraumati- zados com trauma craniencefálico grave traz riscos, particularmente pela possibilidade de falha no diagnóstico de lesões de vísceras ocas. Analisando-se retrospectivamente, o tratamento não operatório das lesões abdominais poderia ter sido empregado em alguns dos doentes de nossa amostra. Contudo, permanece a dúvida quanto a segurança deste procedimento em doentes com diminuição do nível de consciência.

Estudamos apenas os traumatizados com TCE grave, onde o diagnóstico da lesão abdominal é ainda mais difícil e a possibilidade de craniotomia maior. Da nossa população de doentes, $32 \%$ necessitaram de craniotomia e $15 \%$ foram submetidos a laparotomia. Como esperado em vítimas de trauma fechado, o fígado e o baço foram os órgãos mais freqüentemente lesados. Na sua maioria lesões menores e de fácil tratamento. Contudo a falha no diagnóstico destas lesões pode trazer repercussões sérias, e até mesmo levar ao óbito por hemorragia. Considerando que nenhum destes doentes teve o diagnóstico das lesões abdominais somente com o exame físico, justifica-se a avaliação objetiva do abdome com métodos complementares em todos os politraumatizados com TCE grave. A suspeita deve ser ainda maior nos admitidos em choque ou com lesões ortopédicas de tratamento operatório pela alta probabilidade de lesões abdominais associadas. Nos hemodinamicamente instáveis, a lavagem peritoneal diagnóstica ou a ultra-sonografia na sala de emergência são as opções mais rápidas e efetivas ${ }^{2,8,11}$. Nos doentes estáveis hemodinamicamente métodos de imagem podem ser empregados, compreendendo-se suas limitações.

Entretanto, o atraso do tratamento de lesões intracranianas para realização de exames complementares influencia o prognóstico. Nos doentes que necessitam de operação de emergência para o tratamento de lesões cranianas a lavagem peritoneal diagnóstica pode ser realizada na sala de operações, e, se positiva, a laparotomia é indicada.

Portanto, a análise dos dados apresentados sugere que uma parcela considerável dos traumatizados com TCE grave apresenta lesões abdominais, o exame físico contribui pouco para o seu diagnóstico, e, desta forma, justifica-se a investigação objetiva do abdome com métodos complementares em todos estes casos.

\begin{abstract}
Background: The present study was carried out to analyze the abdominal lesions in victims of multiple trauma sustaining severe blunt head trauma. Methods: We retrospectively reviewed charts and trauma protocols of patients admitted from January 1996 to July 1997, who sustained blunt head trauma and underwent head CT Scan. Those admitted with a Glasgow Coma Score $(G C S) \leq 8$ were selected for this study. There were 52 patients who fulfilled the inclusion criteria, 44 male and 8 female, with a mean age of 37 years. Demographic data, mechanism of injury, trauma indices (RTS and ISS), diagnostic exams, treatment and evolution were evaluated. We employed the $X^{2}$ test, Student - $t$ test, and Fisher exact test for the statistical analysis. Results: Pedestrian versus auto was the most frequent mechanism of trauma, occurring in 25 cases. The mean RTS and ISS were 5.18 and 27 respectively. None of these patients had the diagnosis of abdominal lesion based only in physical examination. Forty four were assessed with diagnostic peritoneal lavage and 8 with abdominal ultrasound or computed tomography. Eight (15\%) patients sustained abdominal lesions, including 5 hepatic, 4 splenic and one colonic injury. There were 17 craniotomies and 8 laparotomies (4 non therapeutic). A higher incidence of abdominal lesions was observed in
\end{abstract}


patients sustaining either shock on admission or orthopedic lesions requiring surgical repair. The mortality was $71 \%$. Conclusion: Considering the incidence of abdominal lesions and the difficulties for their diagnosis in those patients sustaining severe head trauma, the objective assessment of abdomen by complementary methods is advisable in all cases.

Key words: Brain injury; Abdominal trauma; Laparotomy; Diagnostic; Head trauma.

\section{BIBLIOGRAFIA}

1. Gennarelli TA, Champion HR, Sacco WJ, et al. - Mortality of patients with head injury and extracranial injury in trauma centers. J. Trauma, 1989; 29: 1193-1202.

2. Trunkey DD, Hill AC, Schecter WP. "Abdominal trauma and indications for celiotomy". In Moore EE, Mattox KL, Feliciano DV (eds): Trauma. $2^{\text {nd }}$ Edition . Connecticut: Apleton e Lange, 1991; pp 409-426.

3. Powell DC, Bivings BA, Bell RM - Diagnostic Peritoneal Lavage. Surg. Gynecol. Obst., 1982; 155: 257-264.

4. Wisner DH, Victor NS, Holcroft JW - Priorities in the management of multiple trauma: intracranial versus intraabdominal injury. J. Trauma, 1993; 35: 271-276.

5. Thomason M, Mesik J, Rutledge R et al. - Head CT scanning versus urgent exploration in the hypotensive blunt trauma patient. J Trauma, 1993; 34: 40-45.

6. Mackersie RC, Tiwary AD, Shackford SR et al. - Intra-abdominal injury following blunt trauma. Arch Surg, 1989; 124: 809-813.

7. West JG, Trunkey DD, West JG et al. - Systems of trauma care: a study of two countries. Arch Surg, 1979; 114: 455-460.

8. Fabian TC, Croce MA. "Abdominal trauma, including indications for celiotomy". In Feliciano D, Moore EE, Mattox K (eds): Trauma. $3^{\text {nd }}$ Edition. Stamford, Connecticut: Apleton and Lange, 1996; pp 441-460.

9. Winchell RJ, Hoyt DB, Simons RK - Use of computed tomography of the head in the hypotensive blunt trauma patient. Ann Emerg Med, 1995; 25: 737-742.

10. Branney SW, Moore EE, Cantrill SV et al. - Ultrasound based key clinical pathway reduces the use hospital resources for the evaluation of blunt abdominal trauma. J Trauma, 1997; 42: 1086-1090.

11. Chiu WC, Cushing BM, Rodriguez A et al. - Abdominal injuries without hemoperitoneum: a potential limitation of focused abdominal sonography for trauma. J Trauma, 1997; 42: 617-623.

12. The abbreviated injury scale. 1990 revision. Association for advancement of automotive medicine. 190, Des Plaines, IL, USA.

13. Moore EE, Gogbill T, Malangoni M et al. - Escalas de Lesão Orgânica. Clín Cir Am N, 1995; 2: 297-308.

14. Valadka AB, Narayan RK. "Injury to the cranium". In Feliciano D, Moore EE, Mattox K (eds): Trauma. $3^{\text {rd }}$ Edition. Stamford, Connecticut. Apleton and Lange, 1996; pag 267-278.
15. Champion HR, Sacco WJ, Copes W et al. - A revision of the trauma score. J Trauma, 1989; 29: 623-629.

16. Baker S, O’Neil B, Haddon W et al. - The Injury Severity Score: A method for describing patients with multiple injuries and evaluating emergency care. J Trauma, 1974; 14: 187-196.

17. Boyd CR, Tolson MA, Copes WS - Evaluating trauma care: The TRISS method. J Trauma, 1987; 27: 370-377.

18. Mendelow AD, Kami MZ, Paul KS et al.- Extradural hematoma: Effect of delayed treatment. Br J Med, 1979; $1: 1240-1242$

19. Seelig JM, Becker DP, Miller JD et al. - Traumatic acute subdural hematoma: Major mortality reduction in comatose patients treated within four hours. N Engl $\mathbf{J}$ Med, 1981; 304: 1511-1518.

20. Renz BM, Feliciano D - Unnecessary laparotomies for trauma: a prospective study of morbidity. J Trauma, 1995; 38: 350-356.

21. Gutman MB, Moulton RJ, Sullivan MA et al. - Relative incidence of intracranial mass lesions and severe torso injury after accidental injury: implication for triage and management. J Trauma, 1991; 31(7): 974-977.

22. Archer LP, Rogers FB, Schackford SR - Selective nonoperative management of liver and spleen injuries in neurologically impaired patients. Arch Surg, 1996; 131: 309-315.

Endereço para Comunicação:

José G. Parreira

Rua Cesário Motta Jr., 167, apartamento 83.

Santa Cecília, São Paulo (SP). CEP: 01238-010.

email:jgparreira@uol.com.br 